\title{
Wideband Gain Flattened Hybrid Erbium-doped Fiber Amplifier/Fiber Raman Amplifier
}

\author{
Hossein Afkhami \\ Islamic Azad University, Sepidan Branch, Sepidan, Shiraz, Iran \\ Alireza Mowla and Nosrat Granpayeh* \\ Faculty of Electrical and Computer Engineering, K. N. Toosi University of Technology, \\ Tehran 1431714191, Iran \\ Azadeh Rastegari Hormozi \\ Islamic Azad University, Arsanjan Branch, Arsanjan, Shiraz, Iran
}

(Received September 29, 2010 : revised November 29, 2010 : accepted November 29, 2010)

\begin{abstract}
An optimal wideband gain flattened hybrid erbium-doped fiber amplifier/fiber Raman amplifier (EDFA/FRA) has been introduced. A new and effective optimization method called particle swarm optimization (PSO) is employed to find the optimized parameters of the EDFA/FRA. Numerous parameters which are the parameters of the erbium-doped fiber amplifier (EDFA) and the fiber Raman amplifier (FRA) define the gain spectrum of a hybrid EDFA/FRA. Here, we optimize the length, $\mathrm{Er}^{3+}$ concentration, and $^{3}$ pump power and wavelength of the EDFA and also pump powers and wavelengths of the FRA to obtain the flattest operating gain spectrum. Hybrid EDFA/FRA with 6-pumped- and 10-pumped-FRAs have been studied. Gain spectrum variations are 1.392 and $1.043 \mathrm{~dB}$ for the 6-pumped- and 10-pumped-FRAs, respectively, in the $108.5 \mathrm{~km}$ hybrid EDFA/FRAs, with $1 \mathrm{~mW}$ of input signal powers. Dense wavelength division multiplexing (DWDM) system with 60 signal channels in the wavelength range of 1529.2-1627.1 $\mathrm{nm}$, i.e. the wide bandwidth of $98 \mathrm{~nm}$, is studied. In this work, we have added FRA's pump wavelengths to the optimization parameters to obtain better results in comparison with the results presented in our previous works.
\end{abstract}

Keywords: Hybrid fiber amplifier, Erbium-doped fiber amplifier, Fiber Raman amplifier, Optimization, Particle swarm optimization

OCIS codes : (060.2320) Fiber optics amplifiers and oscillators; (140.4480) Optical amplifiers; (060.0060) Fiber optics and optical communications; (060.2410) Fibers, erbium

\section{INTRODUCTION}

After the invention of EDFAs, data transfer capacities of optical communication systems exploded based on wavelength division multiplexing (WDM) systems [1]. Then more demands for higher capacity systems, in addition to the progress in optical device technology, encouraged the researchers to focus on the multi-wavelength pump FRAs which have broader bandwidths [2]. Hybrid EDFA/FRAs could have the benefits of both EDFAs and FRAs, hence some efforts have been carried on to offer efficient constructions for hybrid EDFA/FRAs [3]. Hybrid EDFA/FRAs have long lengths and consequently lower numbers of link repeaters are needed. To build these hybrid amplifiers, low price EDFAs are used. Their costs are relatively lower than FRAs and their noise figures (NFs) are lower than those of the EDFAs [2]. A WDM system with hybrid EDFA/ FRAs has lots of elements and the combination of these elements and the value of their parameters are of great importance. The module combination is the art of engineering work. But choosing the module parameters could be difficult, because these parameters affect the characteristics of the hybrid EDFA/FRAs. Here, a global optimization method (GOM) is quite necessary. GOMs help us to find the optimum

\footnotetext{
*Corresponding author: granpayeh@eetd.kntu.ac.ir

Color versions of one or more of the figures in this paper are available online.
} 
values for parameters of the modules. PSO is a GOM that is effective and fast and can deal with a high number of variables $[4,5]$. Using PSO, the parameters of the EDFA and FRA used in hybrid EDFA/FRA have been optimized to obtain a wideband gain flattened amplifier. In this paper, hybrid EDFA/FRA configurations are introduced, the method of simulation of hybrid EDFA/FRA is discussed and then PSO is presented and the results of optimization are given for different pumping schemes and configurations. In addition to optimization of the length, $\mathrm{Er}^{3+}$ concentration, and pump power and wavelength of the EDFA and also pump powers of the FRA all of which have been carried on in our previous work [6], the pump wavelengths of the FRA are optimized in order to have a flatter-gain hybrid EDFA/FRA. Adding the pump wavelengths of the FRA to the other optimization variables increases the number of optimization variables to 24 in the 10 pumped FRA hybrid EDFA/FRA. Dealing with this high number of variables is quite a challenge. Here PSO shows its strength to manipulate a large number of variables in a fast and straightforward manner in comparison with the other optimization methods such as genetic algorithm (GA) [7-14]. The results are discussed in section $\mathrm{V}$.

\section{HYBRID EDFA/FRA CONFIGURATION}

FIG. 1 depicts the configuration which we have chosen for the hybrid EDFA/FRA. There are 60 signal channels with frequencies between 184.25 and $196.05 \mathrm{THz}$, placed equally in $200 \mathrm{GHz}$ of frequency distances launched to the single mode fiber (SMF). SMF allows the propagation of only one mode, retains the fidelity of each light ray and has higher bandwidths over longer links. TABLE 1 presents the characteristics of the designed WDM system. If we utilize communication systems with the rate of $40 \mathrm{Gbit} / \mathrm{s}$ at each of these channels, the capacity of this WDM link could reach $2.4 \mathrm{Tbit} / \mathrm{s}$. Choosing the values of the input signal powers is of great importance and will affect the gain spectrum variations of the amplifier. We select the input signal powers to be 1 or $10 \mathrm{~mW}$ to have a comparison

One span of the WDM system

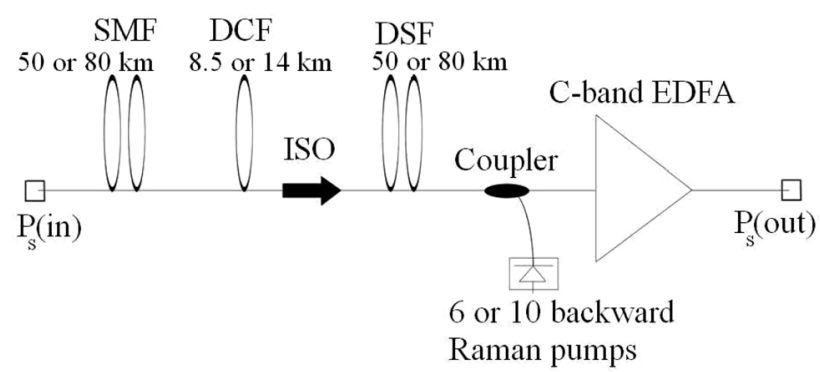

FIG. 1. Configurations of the hybrid EDFA/FRA with 6- or 10-pumped-FRA in backward direction. between the results. Here, SMF acts as the transmission medium. A dispersion compensating fiber (DCF) is located after the SMF to compensate the dispersion that took place in the SMF. DCF has a high negative value of dispersion which is supposed to neutralize the SMF lower positive value of dispersion. So, shorter length of DCF compared to the length of SMF is needed and the length of DCF must be chosen appropriately. Afterward, a dispersion shifted fiber (DSF) is placed which has zero dispersion at $1550 \mathrm{~nm}$ wavelength and is pumped by 6 or 10 backward pump LD sources which acts as an FRA. FRA pump powers are propagated counter to the direction of the signal powers. In this paper, we have chosen 2 different sets of fiber lengths. Accordingly, SMF, DCF, and DSF could be $50,8.5$, and $50 \mathrm{~km}$ in the first configuration and 80,14 , and $80 \mathrm{~km}$ in the second configuration, respectively. An isolator (ISO) is laid between the DCF and DSF to suppress the residue of the FRA backward pumps and noise, raised in the DSF. A WDM coupler is set up to couple the power of pumps to the DSF in the counter-propagating direction. Pump powers and wavelengths are chosen through the optimization process discussed in section IV. Next module is a C-band EDFA. Principal range of amplification in the C-band EDFA is 1530-1570 nm and its gain falls at wavelengths over $1570 \mathrm{~nm}$. On the other hand, the amplification at the higher range of 1570-1630 takes place mostly through the DSF as an FRA. TABLE 2 lists the characteristics of different modules used in our hybrid EDFA/FRA configurations. Lots of variables exist in the design of EDFA. In this paper we have chosen 4 of the most important variables to be optimized, the length, the $\mathrm{Er}^{3+}$ concentration, the pump power and wavelength of the EDFA. Finally, we have a detector at the end of the link span to receive the output powers. Gain spectrum variations will be computed at this point. This span can be repeated if longer links are needed. As indicated, four different configurations have been proposed. These configurations are 108.5 or $174 \mathrm{~km}$ in length and each could adopt 6- or 10-pumped-FRA. Additionally, the amplifier characteristics are calculated in the cases of different input signal powers of 1 or $10 \mathrm{~mW}$ for each channel. As discussed in section $\mathrm{V}$, gain spectrum variations of $1.794 \mathrm{~dB}$ can be achieved for the $174 \mathrm{~km}$ link span, 10-pumped-FRA hybrid EDFA/FRA with $1 \mathrm{~mW}$ of input signal power.

TABLE 1. Characteristics of the WDM transmission system

\begin{tabular}{c|c}
\hline \hline WDM characteristic & Value \\
\hline Number of signal channels & 60 \\
\hline Signal wavelength range & $1529.2-1627.1 \mathrm{~nm}$ \\
\hline Signal channels start frequency & $184.25 \mathrm{THz}$ \\
\hline Signal channels end frequency & $196.05 \mathrm{THz}$ \\
\hline Signal channels frequency space & $0.2 \mathrm{THz}$ \\
\hline Raman pumps wavelength range & $1420-1520 \mathrm{~nm}$ \\
\hline
\end{tabular}


TABLE 2. Characteristics of hybrid EDFA/FRA fiber modules

\begin{tabular}{c|c|c|c|c}
\hline \hline Module & Fiber type & Attenuation $(\mathrm{dB} / \mathrm{km})$ & Dispersion $(\mathrm{ps} /(\mathrm{nm} . \mathrm{km}))$ & Length \\
\hline Transmission & SMF & FIG. 2(c) [24] & $16.5[2]$ & 50 and $80 \mathrm{~km}$ \\
\hline Compensating & DCF & FIG. 2(c) [24] & $-95[24]$ & 8.5 and $14 \mathrm{~km}$ \\
\hline FRA & DSF & FIG. 2(c) [2] & 0 & 50 and $80 \mathrm{~km}$ \\
\hline EDFA & SMF & FIG. 2(c) [24] & 16.5 & Variable (1 to 10 m) \\
\hline
\end{tabular}

TABLE 3. EDFA and FRA parameters used in the hybrid amplifier modeling

\begin{tabular}{c|c|c|c}
\hline \hline FRA parameter & Value & EDFA parameter & Value \\
\hline Rayleigh backscattering $\gamma\left(\mathrm{m}^{-1}\right)$ & $7 \times 10^{-8}$ & Upper level life time & $10 \mathrm{~ms}$ \\
\hline Temperature $T\left({ }^{\circ} \mathrm{K}\right)$ & 300 & Cladding index & 1.445 \\
\hline Number of pumps & 6 or 10 & Fiber core radius & $6.5 \mu \mathrm{m}$ \\
\hline FRA Pumps direction & Backward & Excess fiber loss & $0.03 \mathrm{~dB} / \mathrm{m}$ \\
\hline
\end{tabular}

\section{HYBRID EDFA/FRA SIMULATION}

Simulation of EDFA and FRA should be done separately. Also, attenuation, dispersion and noise of the other components such as SMF, DCF, DSF, erbium-doped fiber (EDF), and ISO must be taken into account. Therefore, the simulation of hybrid EDFA/FRA has to be a combination of simulations of different modules and components of the hybrid amplifier. In the following sections we discuss the simulation of EDFAs and FRAs separately.

1. Simulation of the EDFA: Giles model [15] is the most common model used for the optical amplification that occurs in a silica fiber doped by $\mathrm{Er}^{3+}$. We assume a two-level, homogeneously broadened model for $\mathrm{Er}^{3+}$ that is sufficient to have a simple and accurate EDFA model $[15,16]$. In this model ${ }^{4} \mathrm{I}_{15 / 2}$ is the ground level and ${ }^{4} \mathrm{I}_{13 / 2}$ is the excited level. We suppose that all the radiative transitions occur between these two levels. Computer analysis and simulation is discrete, so we should break the continuous spectrum of the optical waves into discrete optical beams with frequency bandwidth of $\Delta v_{k}$ and optical power of $P_{k}$ centered at frequency $v_{k}$ or wavelength $\lambda_{k}$. We should solve an ordinary differential equation for each one of the optical beams to find the propagation of optical powers along the fiber. Therefore, $N$ ordinary differential equations should be solved, where $N$ is the number of the pump and signal channels. Rate equations of the excited level of $\mathrm{Er}^{3+}$ ions are computed as a summation of the emission and absorption contributions of all the $N$ optical beams [17]. We have used $\mathrm{Al} / \mathrm{P}$-silica fiber as the EDFA, the emission and absorption cross sections of which are depicted in FIG. 2(a) [18]. All fibers are assumed to be SMFs, hence the $V$ number should be less than 2.405 . Also we have assumed the steady state condition of $\partial N_{2}(r, z, t) / \partial t=0$ where $N_{2}$ is the upper level population, and also the weakly guiding approximation of $\Delta=\left(n_{1}-n_{2}\right) / n_{2}<<1$ where $n_{1}$ and $n_{2}$ are the refractive indices of the fiber core and cladding, respectively. The flow of signal and pump power through EDFA is formulated by ordinary differential equations [17]. Rate equations in the steady-state condition have to be solved to acquire the metastable level population of $\mathrm{Er}^{3+}$ ions [17].

Since the EDFA pump is in the co-propagating direction, we need to solve an initial value problem. We ignore the counter propagating pumps and eliminate the backward amplified spontaneous emission (ASE) from our simulation to avoid solving the boundary value problem which is a bit more challenging. In this paper, a one-step predictorcorrector method [19] is used to simulate the EDFA. Using this method, the propagated powers at channels are calculated step by step using their values at the previous steps and their predicted values at the next steps [20].

The pumping wavelength is around $1480 \mathrm{~nm}$. Step index fiber with uniform distribution of $\mathrm{Er}^{3+}$ ions are used in this simulation. EDFA characteristics which have been used are listed in TABLE 3.

2. Simulation of the FRA: Coupled ordinary differential equations govern the propagation of the average optical power of pumps, signals, ASEs, and Rayleigh backscattering beams in a FRA $[19,21]$. For co-propagating pumps, an initial value problem must be solved and for the case of counter-propagating or bidirectional pumps, two-point boundary value problems must be solved. Here, we have counterpropagating pumps, hence a two-point boundary value problem has to be solved. Four-step predictor-corrector method based on the Adams-Bashforth-Moulton formula [19] and shooting algorithm [22] are used to solve this problem numerically [23]. FIG. 2(b) shows the Raman gain efficiency of the DSF [2] and FIG. 2(c) shows attenuation spectra of the DSF [2], SMF, and DCF [24] used in our simulation.

We have used $\mathrm{C}^{+}$programming to model the hybrid EDFA/ FRA. The time needed to accomplish the simulation, depends 


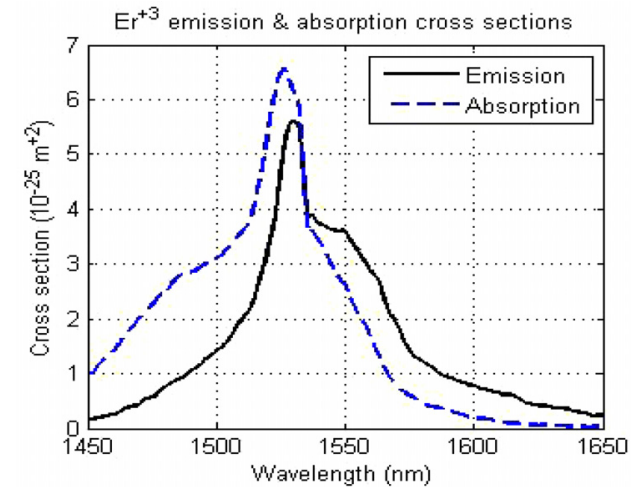

(a)

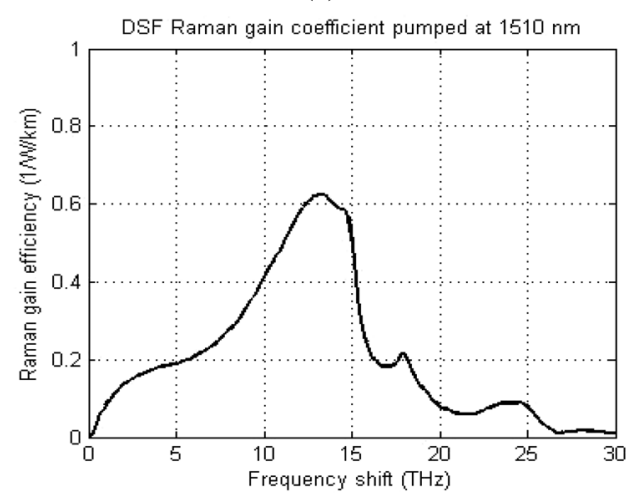

(b)

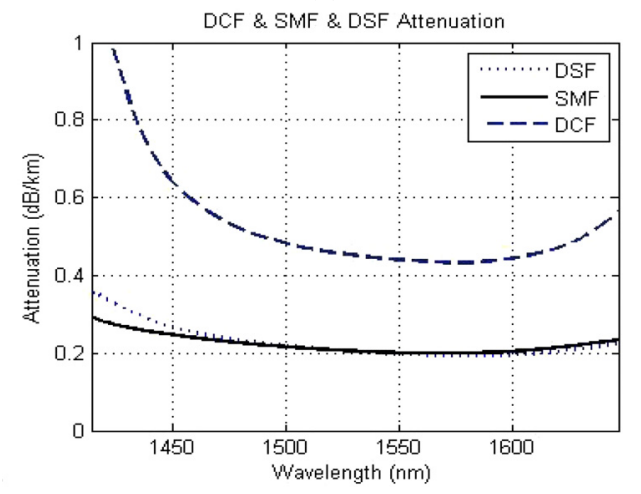

(c)

FIG. 2. Characteristics of the fibers (a) emission and absorption cross sections of $\mathrm{Er}^{+3}$ doped in Al/P-silica SMF (b) Raman gain efficiency of DSF (c) DCF, SMF, and DSF attenuation spectrum. [2, 23, 24]

on the configuration of the setup. It could be 0.2 to 1 second when run by an AMD Athlon 2.71 GHz CPU with $1.00 \mathrm{~Gb}$ of RAM.

\section{PARTICLE SWARM OPTIMIZATION IMPLEMENTATION}

PSO is a population-based stochastic method for solving optimization problems which is classified as a swarm intelligence technique. In this method, a population of particles which are bunches of variables that present candidate solutions, will be selected randomly from the possible solution space and these particles will move according to their social learning from each other toward the optimum solution. The rules and constraints which lead the flying particles in the search space toward the global optimized solution are originated from the behavioral models of bird flocks [4, 5]. This method was introduced and developed by Kennedy and Eberhart [25, 26].

Let us assume that $F$ is the fitness function. There are $n$ particles $P_{i}$ where $i=1, \cdots, n, x_{i}$ is the current position of $P_{i}, G b$ is the global best which is the best fitness value that has ever happened, $P b_{i}$ is the personal best of $P_{i}$ which is the best previous fitness value of $P_{i}, x_{G b}$ and $x_{P b i}$ are the positions of the $G b$ and $P b_{i}$, respectively, $v_{i}$ is the velocity of $P_{i}$ and $k$ is the time interval. We can present PSO method in the following steps:

Step 1. $P_{i s}$ are randomly chosen from the search space to form the swarm $P(k)$.

Step 2. Calculate the fitness values $F\left(x_{i}(k)\right)$ for each $P_{i}$. Step 3. If $F\left(x_{i}(k)\right)>P b_{i}$, then $P b_{i}=F\left(x_{i}(k)\right)$ and $x_{P b i}=x_{i}(k)$.

Step 4. If $F\left(x_{i}(k)\right)_{\text {max }}>G b$, then $G b=F\left(x_{i}(k)\right)_{\text {max }}$ and $x_{G b}=x_{i}(k)$.

Step 5. Set the new velocities

$$
\begin{gathered}
v_{i}(k)=\varphi v_{i}(k-1)+\rho_{1} C_{f}\left(x_{P b i}-\right. \\
\left.x_{i}(k)\right)+\rho_{2} C_{f}\left(x_{G b}-x_{i}(k)\right) \\
\varphi=1-0.6 \frac{1-k}{1-k_{\max }}
\end{gathered}
$$

where $\varphi$ is the inertia weight, $k$ is the iteration number and $k_{\max }=300$ is the total number of iterations, $C_{f}=2$ is the accelerator constant. $\rho_{1}$ and $\rho_{2}$ are uniform random values between 0 and 1.

Step 6. Set the positions

$$
x_{i}(k)=x_{i}(k-1)+v_{i}(k) \quad(k=k+1)
$$

Step 7. Check the convergence criterion, if fulfilled, the optimization is over, if not go to step 2.

To perform the optimization, PSO acts as the main program and the simulation of the hybrid EDFA/FRA will be the subprogram. Fitness function is the maximum signal network gain deviation from zero which is supposed to be minimum, formulated in the following:

$$
F_{f i t}=\operatorname{Max}\left|10 \times \log \left(\frac{P_{s}^{k}(L)}{P_{s}^{k}(0)}\right)\right|
$$

where $P_{s}^{k}(0)$ and $P_{s}^{k}(L)$ are the $k$-th input and output signal powers, respectively. Taking into account the design 
TABLE 4. Variables of the optimization of the EDFA/FRAs and their ranges

\begin{tabular}{c|c}
\hline \hline Variable to be optimized & Range \\
\hline EDFA pumping wavelength $\lambda_{p}$ & $1450-1500 \mathrm{~nm}$ \\
\hline EDFA Er $^{3+}$ concentration $n_{t}$ & $(1-30) \times 10^{24}$ \\
\hline EDFA fiber length $L$ & $1-10 \mathrm{~m}$ \\
\hline EDFA pump power & $100-800 \mathrm{~mW}$ \\
\hline FRA pump wavelengths & $1420-1520 \mathrm{~nm}$ \\
\hline FRA pump powers & $5-600 \mathrm{~mW}$ \\
\hline
\end{tabular}

criteria, variables of optimization and their ranges in this work have been listed in TABLE 4. As it was mentioned, we used $\mathrm{C}^{++}$programming to simulate the hybrid EDFA/ FRA and execute the optimization process. The necessary time to accomplish one round of optimization depends on the configuration which is to be optimized and could be between 2 and 3 hours, by an AMD Athlon $2.71 \mathrm{GHz}$ CPU with $1.00 \mathrm{~Gb}$ of RAM. The number of particles and iterations in our PSO model are 40 and 300, respectively.

\section{RESULTS AND DISCUSSIONS}

In this paper we have simulated 4 different configurations for hybrid EDFA/FRA. Two configurations for 108.5 $\mathrm{km}$ and two for $174 \mathrm{~km}$ link with 6- and 10-pumped-FRA. Also, we survey the results for both input signal powers of 1 or $10 \mathrm{~mW}$.

A group of optimization variables or the group of simulation inputs which are introduced in TABLE 4 are known as a particle $P_{i}$ in the PSO. We have $n=40$ particles in our optimization process and it means that we have 40 groups of optimization variables which are randomly spread in our optimization space. Each one of the optimization variables makes a dimension of the optimization space. Particle positions $x_{i}$ are arrays which are composed of the optimization variable values. We also have $n=40$ particle positions $x_{\mathrm{i}}$ in our optimization process that need to be optimized. At each iteration, we insert the inputs which are the optimization variable values or the particle positions to the main hybrid EDFA/FRA simulation computer program or fitness function. Then the simulation will result in the output or the fitness value $F\left(x_{\mathrm{i}}\right)$ which is the maximum signal network gain deviation from zero. We consider the deviation from zero to take into account both the negative and positive gain values. According to the PSO steps which are introduced in the previous section, new values for optimization variables are selected at the end of each iteration. We have 24 and 16 optimization variables for 10- and 6- pumped FRA hybrid amplifiers. So, the particle positions are composed of 24 or 16 elements.

To reach the flattest gain spectrum for the hybrid EDFA/FRA, it is better to set the EDFA pump wavelength to have a C-band EDFA. Because of the shape of the EDFA gain spectrum, it is preferred to have amplification at lower wavelengths by EDFA and at higher wavelengths by FRA. In the optimization process, the EDFA pump power is inclined to attain high values. So, it is better to choose $1480 \mathrm{~nm}$ pumping wavelength for the EDFA, although it has higher noise compared to $980 \mathrm{~nm}$ ones. Also, in high power schemes it is more efficient to use $1480 \mathrm{~nm}$ pumping wavelength. The radius of the $\mathrm{Er}^{3+}$ doped area at EDFA is equal to the radius of the fiber used as EDFA that is $6.5 \mu \mathrm{m}$. An EDFA with a small core radius will result in better gain spectrum characteristics, but its fabrication is hard and it is more susceptible to damage in facing high EDFA pump power. Also a small core radius EDFA is rare in the market. $\mathrm{Er}^{3+}$ ion concentration has to be chosen in a confined area, since low $\mathrm{Er}^{3+}$ concentration would elongate the EDFA and consequently create higher variation of the gain spectrum. Also, high $\mathrm{Er}^{3+}$ concentration will increase unfavorable intra- ion interactions [1]. The main source of noise of EDFAs is ASE. The level of noise in FRAs is lower than that in EDFAs. In this paper, we have assumed an acceptably low level for noise, and to speed up the optimization process, we have not simulated noises. Lower input signal power in EDFAs will result in higher and flatter gain spectra, but input signal power should not be so low that it could not be retrieved from the noise. The designed amplifier is a distributed amplifier, and it is better to define net gain for it. We prefer the signal powers at the end of the link to be the same as their values at the beginning of the link. So, the ideal net gain of our hybrid EDFA/FRA is zero. We have assumed the EDFA to be polarization independent as it almost is, but we consider the polarization dependency of FRA by including a factor in our simulations.

Amplification in the FRA is due to the nonlinear phenomenon of stimulated Raman scattering (SRS). By using a high number of pumps in FRAs with different wavelengths and powers, the gain spectrum is tailored and the EDFA gain variation is compensated. The amplification band can be chosen simply by adjusting the pump wavelengths at appropriate distances. The noise transition from pumps to signals occurs less in the counter-propagating pump scheme, hence it is more advantageous to place the pumps at the end of the FRA and increase the length of link span. We have to appoint the input signal powers to somehow avoid harmful interference with noise due to the low level of the input signal power, and unfavorable nonlinear effects due to the high level of the input signal powers.

All the optimization results, obtained by PSO, for different schemes and configurations are given in TABLES 5-8. It is obvious that gain spectrum variations will be lower if we choose 10-pumped configurations instead of 6-pumped ones, or if we choose $108.5 \mathrm{~km}$ links instead of $174 \mathrm{~km}$ ones, and also if we choose $1 \mathrm{~mW}$ of input signal power instead of $10 \mathrm{~mW}$. As listed in TABLE 5, gain spectrum variations of 1.392 and $1.043 \mathrm{~dB}$ are attained for 108.5 
Wideband Gain Flattened Hybrid Erbium-doped Fiber Amplifier/Fiber Raman Amplifier - Hossein Afkhami et al. 347

TABLE 5. Values of the optimized parameters of 6- \& 10-pumped-FRA, $108.5 \mathrm{~km}$ hybrid EDFA/FRA with $1 \mathrm{~mW}$ of input signal power, obtained by PSO

\begin{tabular}{c|c|c}
\hline \hline Variable & Value for 10 pumps FRA HA & Value for 6 pumps FRA HA \\
\hline EDFA pump wavelength $\lambda_{p}$ & $1478.9 \mathrm{~nm}$ & $1460.4 \mathrm{~nm}$ \\
\hline EDFA Er ${ }^{3+}$ concentration $n_{t}$ & $7.2 \times 10^{24}$ & $8.4 \times 10^{24}$ \\
\hline EDFA fiber length $L$ & $1.139 \mathrm{~m}$ & $1.247 \mathrm{~m}$ \\
\hline EDFA pump power & $338.5 \mathrm{~mW}$ & $283.6 \mathrm{~mW}$ \\
\hline FRA pump power $P_{1}$ and wavelength $\lambda_{1}$ & $114.2 \mathrm{~mW}$ and $1420.9 \mathrm{~nm}$ & $102.4 \mathrm{~mW}$ and $1430.3 \mathrm{~nm}$ \\
\hline FRA pump power $P_{2}$ and wavelength $\lambda_{2}$ & $261.2 \mathrm{~mW}$ and $1431.5 \mathrm{~nm}$ & $350.5 \mathrm{~mW}$ and $1435.8 \mathrm{~nm}$ \\
\hline FRA pump power $P_{3}$ and wavelength $\lambda_{3}$ & $177.3 \mathrm{~mW}$ and $1440.5 \mathrm{~nm}$ & $18.6 \mathrm{~mW}$ and $1467.8 \mathrm{~nm}$ \\
\hline FRA pump power $P_{4}$ and wavelength $\lambda_{4}$ & $15.9 \mathrm{~mW}$ and $1458.2 \mathrm{~nm}$ & $117.9 \mathrm{~mW}$ and $1476.6 \mathrm{~nm}$ \\
\hline FRA pump power $P_{5}$ and wavelength $\lambda_{5}$ & $64.3 \mathrm{~mW}$ and $1468.1 \mathrm{~nm}$ & $94.0 \mathrm{~mW}$ and $1487.5 \mathrm{~nm}$ \\
\hline FRA pump power $P_{6}$ and wavelength $\lambda_{6}$ & $23.9 \mathrm{~mW}$ and $1476.8 \mathrm{~nm}$ & $74.2 \mathrm{~mW}$ and $1520.9 \mathrm{~nm}$ \\
\hline FRA pump power $P_{7}$ and wavelength $\lambda_{7}$ & $71.4 \mathrm{~mW}$ and $1481.1 \mathrm{~nm}$ & ---- \\
\hline FRA pump power $P_{8}$ and wavelength $\lambda_{8}$ & $24.8 \mathrm{~mW}$ and $1489.5 \mathrm{~nm}$ & ---- \\
\hline FRA pump power $P_{9}$ and wavelength $\lambda_{9}$ & $13.4 \mathrm{~mW}$ and $1500.9 \mathrm{~nm}$ & ---- \\
\hline FRA pump power $P_{10}$ and wavelength $\lambda_{10}$ & $43.6 \mathrm{~mW}$ and $1519.3 \mathrm{~nm}$ & $0.696 \mathrm{~dB}$ \\
\hline Fitness value & $0.521 \mathrm{~dB}$ & $1.392 \mathrm{~dB}$ \\
\hline Gain spectrum variations & $1.043 \mathrm{~dB}$ &
\end{tabular}

TABLE 6. Values of the optimized parameters of 6- \& 10-pumped-FRA, $174 \mathrm{~km}$ hybrid EDFA/FRA with $1 \mathrm{~mW}$ of input signal power, obtained by PSO

\begin{tabular}{c|c|c}
\hline \hline Variable & Value for 10 pumps FRA HA & Value for 6 pumps FRA HA \\
\hline EDFA pump wavelength $\lambda_{p}$ & $1460.4 \mathrm{~nm}$ & $1460.7 \mathrm{~nm}$ \\
\hline EDFA Er3+concentration $n_{t}$ & $10.1 \times 10^{24}$ & $14.7 \times 10^{24}$ \\
\hline EDFA fiber length $L$ & $1.017 \mathrm{~m}$ & $1.012 \mathrm{~m}$ \\
\hline EDFA pump power & $799.9 \mathrm{~mW}$ & $265.1 \mathrm{~mW}$ \\
\hline FRA pump power $P_{1}$ and wavelength $\lambda_{1}$ & $435.6 \mathrm{~mW}$ and $1428.7 \mathrm{~nm}$ & $342.2 \mathrm{~mW}$ and $1431.4 \mathrm{~nm}$ \\
\hline FRA pump power $P_{2}$ and wavelength $\lambda_{2}$ & $307.2 \mathrm{~mW}$ and $1438.9 \mathrm{~nm}$ & $434.9 \mathrm{~mW}$ and $1435.6 \mathrm{~nm}$ \\
\hline FRA pump power $P_{3}$ and wavelength $\lambda_{3}$ & $97.5 \mathrm{~mW}$ and $1442.5 \mathrm{~nm}$ & $115.5 \mathrm{~mW}$ and $1465.5 \mathrm{~nm}$ \\
\hline FRA pump power $P_{4}$ and wavelength $\lambda_{4}$ & $10.7 \mathrm{~mW}$ and $1450.0 \mathrm{~nm}$ & $159.5 \mathrm{~mW}$ and $1478.6 \mathrm{~nm}$ \\
\hline FRA pump power $P_{5}$ and wavelength $\lambda_{5}$ & $7.9 \mathrm{~mW}$ and $1466.1 \mathrm{~nm}$ & $50.2 \mathrm{~mW}$ and $1487.7 \mathrm{~nm}$ \\
\hline FRA pump power $P_{6}$ and wavelength $\lambda_{6}$ & $146.9 \mathrm{~mW}$ and $1469.0 \mathrm{~nm}$ & $30.6 \mathrm{~mW}$ and $1518.1 \mathrm{~nm}$ \\
\hline FRA pump power $P_{7}$ and wavelength $\lambda_{7}$ & $123.0 \mathrm{~mW}$ and $1482.6 \mathrm{~nm}$ & ---- \\
\hline FRA pump power $P_{8}$ and wavelength $\lambda_{8}$ & $16.4 \mathrm{~mW}$ and $1490.8 \mathrm{~nm}$ & ---- \\
\hline FRA pump power $P_{9}$ and wavelength $\lambda_{9}$ & $5.8 \mathrm{~mW}$ and $1501.9 \mathrm{~nm}$ & ---- \\
\hline FRA pump power $P_{10}$ and wavelength $\lambda_{10}$ & $24.1 \mathrm{~mW}$ and $1519.3 \mathrm{~nm}$ & $0.932 \mathrm{~dB}$ \\
\hline Fitness value & $0.897 \mathrm{~dB}$ & $1.864 \mathrm{~dB}$ \\
\hline Gain spectrum variations & $1.794 \mathrm{~dB}$ & \\
\hline
\end{tabular}

km, 6- and 10-pumped-FRA hybrid EDFA/FRA, respectively with $1 \mathrm{~mW}$ input signal powers. Also, as listed in TABLE 6, gain spectrum variations of 1.864 and $1.794 \mathrm{~dB}$ are attained for $174 \mathrm{~km}, 6-$ and 10-pumped-FRA hybrid EDFA/FRA, respectively with $1 \mathrm{~mW}$ input signal powers.
Using the results listed in the TABLES 5 and 6, optimized signal gain spectra of 108.5 and $174 \mathrm{~km} \mathrm{10-pumped-FRA}$ hybrid EDFA/FRA are shown in FIG. 3(a), 108.5 and 174 $\mathrm{km}$ 6-pumped-FRA hybrid EDFA/FRA are depicted in FIG. 3(b), 6- and 10-pumped-FRA $108.5 \mathrm{~km}$ hybrid EDFA/FRA 


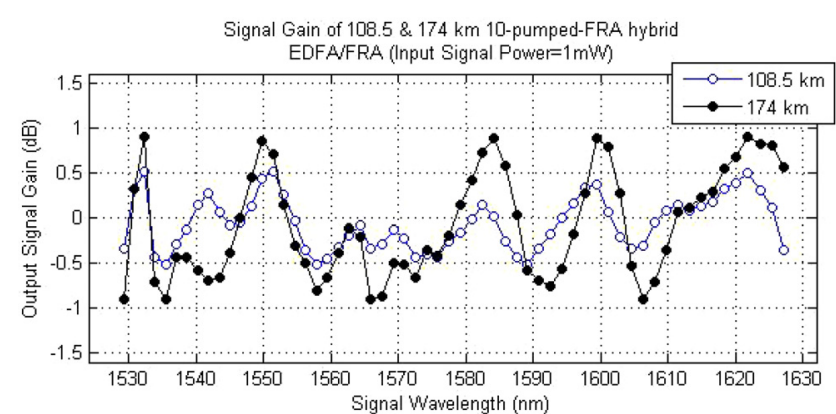

(a)

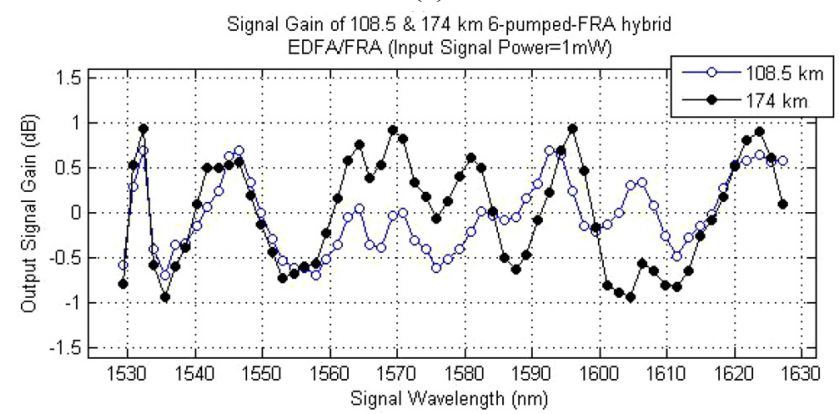

(b)

FIG. 3. Optimum signal gain spectra of (a) $108.5 \& 174 \mathrm{~km}$ 10-pumped-FRA hybrid EDFA/FRA and (b) $108.5 \& 174 \mathrm{~km}$ 6-pumped-FRA hybrid EDFA/FRA (both for input signal power of $1 \mathrm{~mW}$ ).

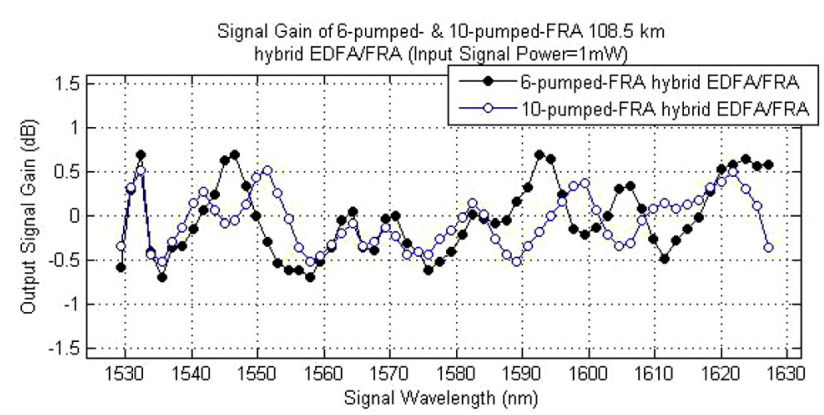

(a)

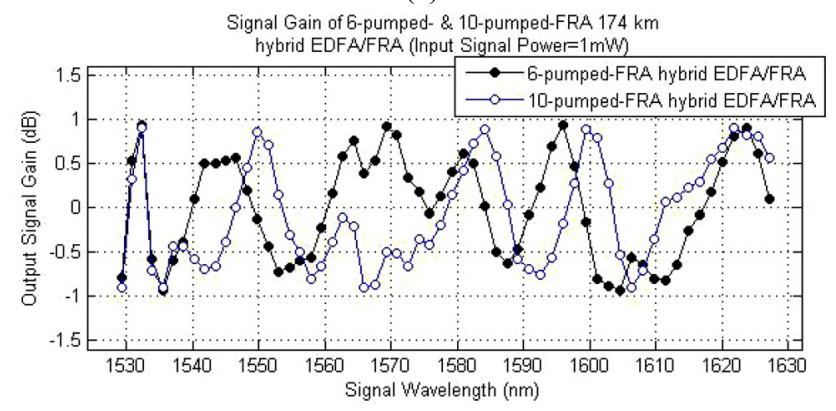

(b)

FIG. 4. Optimum signal gain spectra of (a) 6- \&10-pumpedFRA $108.5 \mathrm{~km}$ hybrid EDFA/FRA and (b) 6- \&10-pumped-FRA $174 \mathrm{~km}$ hybrid EDFA/FRA (both for input signal power of $1 \mathrm{~mW})$.

TABLE 7. Values of the optimized parameters of 6- \& 10-pumped-FRA, $108.5 \mathrm{~km}$ hybrid EDFA/FRA with 10mW of input signal power, obtained by PSO

\begin{tabular}{|c|c|c|}
\hline Variable & Value for 10 pumps FRA HA & Value for 6 pumps FRA HA \\
\hline EDFA pump wavelength $\lambda_{p}$ & $1475.8 \mathrm{~nm}$ & $1478.9 \mathrm{~nm}$ \\
\hline EDFA Er ${ }^{3+}$ concentration $n_{t}$ & $14.1 \times 10^{24}$ & $18.8 \times 10^{24}$ \\
\hline EDFA fiber length $L$ & $1.021 \mathrm{~m}$ & $1.007 \mathrm{~m}$ \\
\hline EDFA pump power & $799.7 \mathrm{~mW}$ & $796.7 \mathrm{~mW}$ \\
\hline FRA pump power $P_{l}$ and wavelength $\lambda_{l}$ & $141.4 \mathrm{~mW}$ and $1422.9 \mathrm{~nm}$ & $329.9 \mathrm{~mW}$ and $1422.6 \mathrm{~nm}$ \\
\hline FRA pump power $P_{2}$ and wavelength $\lambda_{2}$ & $390.0 \mathrm{~mW}$ and $1430.0 \mathrm{~nm}$ & $342.5 \mathrm{~mW}$ and $1434.8 \mathrm{~nm}$ \\
\hline FRA pump power $P_{3}$ and wavelength $\lambda_{3}$ & $205.3 \mathrm{~mW}$ and $1439.5 \mathrm{~nm}$ & $6.1 \mathrm{~mW}$ and $1451.0 \mathrm{~nm}$ \\
\hline FRA pump power $P_{4}$ and wavelength $\lambda_{4}$ & $10.1 \mathrm{~mW}$ and $1449.3 \mathrm{~nm}$ & $98.4 \mathrm{~mW}$ and $1474.9 \mathrm{~nm}$ \\
\hline FRA pump power $P_{5}$ and wavelength $\lambda_{5}$ & $9.3 \mathrm{~mW}$ and $1459.5 \mathrm{~nm}$ & $51.7 \mathrm{~mW}$ and $1487.8 \mathrm{~nm}$ \\
\hline FRA pump power $P_{6}$ and wavelength $\lambda_{6}$ & $12.4 \mathrm{~mW}$ and $1469.5 \mathrm{~nm}$ & $43.3 \mathrm{~mW}$ and $1515.6 \mathrm{~nm}$ \\
\hline FRA pump power $P_{7}$ and wavelength $\lambda_{7}$ & $104.9 \mathrm{~mW}$ and $1478.9 \mathrm{~nm}$ & ----- \\
\hline FRA pump power $P_{8}$ and wavelength $\lambda_{8}$ & $33.1 \mathrm{~mW}$ and $1489.1 \mathrm{~nm}$ & ----- \\
\hline FRA pump power $P_{9}$ and wavelength $\lambda_{9}$ & $11.5 \mathrm{~mW}$ and $1509.1 \mathrm{~nm}$ & ----- \\
\hline FRA pump power $P_{10}$ and wavelength $\lambda_{10}$ & $21.2 \mathrm{~mW}$ and $1519.2 \mathrm{~nm}$ & ---- \\
\hline Fitness value & $0.691 \mathrm{~dB}$ & $0.863 \mathrm{~dB}$ \\
\hline Gain spectrum variations & $1.382 \mathrm{~dB}$ & $1.717 \mathrm{~dB}$ \\
\hline
\end{tabular}

is pictured in FIG. 4(a), and 6- and 10-pumped-FRA 174 $\mathrm{km}$ hybrid EDFA/FRA is pictured in FIG. 4(b), all for 1 $\mathrm{mW}$ input signal powers. These figures present good visual comparison of the optimized gain spectra of different schemes.

It is common to select the FRA pump wavelengths in an equidistant form, but here we search the FRA pump 
Wideband Gain Flattened Hybrid Erbium-doped Fiber Amplifier/Fiber Raman Amplifier - Hossein Afkhami et al. 349

TABLE 8. Values of the optimized parameters of 6- \& 10-pumped-FRA, $174 \mathrm{~km}$ hybrid EDFA/FRA with 10mW of input signal power, obtained by PSO

\begin{tabular}{|c|c|c|}
\hline Variable & Value for 10 pumps FRA HA & Value for 6 pumps FRA HA \\
\hline EDFA pump wavelength $\lambda_{p}$ & $1476.6 \mathrm{~nm}$ & $1476.2 \mathrm{~nm}$ \\
\hline EDFA Er3+concentration $n_{t}$ & $17.8 \times 10^{24}$ & $8.3 \times 10^{24}$ \\
\hline EDFA fiber length $L$ & $1.217 \mathrm{~m}$ & $2.788 \mathrm{~m}$ \\
\hline EDFA pump power & $799.9 \mathrm{~mW}$ & $798.9 \mathrm{~mW}$ \\
\hline FRA pump power $P_{l}$ and wavelength $\lambda_{l}$ & $560.7 \mathrm{~mW}$ and $1428.1 \mathrm{~nm}$ & $559.0 \mathrm{~mW}$ and $1428.1 \mathrm{~nm}$ \\
\hline FRA pump power $P_{2}$ and wavelength $\lambda_{2}$ & $303.3 \mathrm{~mW}$ and $1432.1 \mathrm{~nm}$ & $468.5 \mathrm{~mW}$ and $1435.5 \mathrm{~nm}$ \\
\hline FRA pump power $P_{3}$ and wavelength $\lambda_{3}$ & $173.8 \mathrm{~mW}$ and $1439.5 \mathrm{~nm}$ & $26.5 \mathrm{~mW}$ and $1467.7 \mathrm{~nm}$ \\
\hline FRA pump power $P_{4}$ and wavelength $\lambda_{4}$ & $7.4 \mathrm{~mW}$ and $1449.2 \mathrm{~nm}$ & $118.1 \mathrm{~mW}$ and $1475.6 \mathrm{~nm}$ \\
\hline FRA pump power $P_{5}$ and wavelength $\lambda_{5}$ & $65.4 \mathrm{~mW}$ and $1468.3 \mathrm{~nm}$ & $54.4 \mathrm{~mW}$ and $1487.3 \mathrm{~nm}$ \\
\hline FRA pump power $P_{6}$ and wavelength $\lambda_{6}$ & $104.5 \mathrm{~mW}$ and $1478.5 \mathrm{~nm}$ & $14.9 \mathrm{~mW}$ and $1516.8 \mathrm{~nm}$ \\
\hline FRA pump power $P_{7}$ and wavelength $\lambda_{7}$ & $7.2 \mathrm{~mW}$ and $1487.8 \mathrm{~nm}$ & ----- \\
\hline FRA pump power $P_{8}$ and wavelength $\lambda_{8}$ & $15.0 \mathrm{~mW}$ and $1489.1 \mathrm{~nm}$ & ----- \\
\hline FRA pump power $P_{9}$ and wavelength $\lambda_{9}$ & $8.5 \mathrm{~mW}$ and $1508.2 \mathrm{~nm}$ & $\begin{array}{ll}---- \\
---\end{array}$ \\
\hline FRA pump power $P_{10}$ and wavelength $\lambda_{10}$ & $10.4 \mathrm{~mW}$ and $1516.7 \mathrm{~nm}$ & ----- \\
\hline Fitness value & $1.011 \mathrm{~dB}$ & $1.064 \mathrm{~dB}$ \\
\hline Gain spectrum variations & $2.021 \mathrm{~dB}$ & $2.127 \mathrm{~dB}$ \\
\hline
\end{tabular}

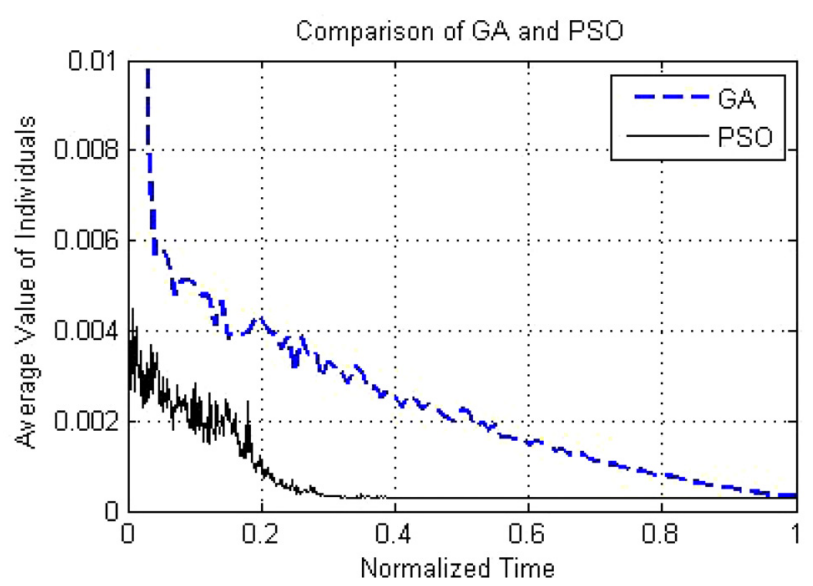

FIG. 5. Comparison of the convergence speeds of the PSO and GA toward the optimum values versus the normalized executing time.

wavelengths through an optimization process which will result in better outcomes for gain spectrum variations. For example, gain spectrum variations of $2.91 \mathrm{~dB}$ for a 130.5 $\mathrm{km}$, 6-pumped-FRA hybrid EDFA/FRA, equidistant FRA pump wavelengths [6] has become $1.864 \mathrm{~dB}$ for $174 \mathrm{~km}$, 6-pumped-FRA hybrid EDFA/FRA, by optimization of FRA pump wavelengths and also gain spectrum variations of $2.03 \mathrm{~dB}$ for a $130.5 \mathrm{~km}, 10$-pumped-FRA hybrid EDFA/ FRA, by equidistant FRA pump wavelengths [6] has become $1.794 \mathrm{~dB}$ for $174 \mathrm{~km}, 10$-pumped-FRA hybrid EDFA/FRA, by optimization of FRA pump wavelengths, which are notable improvements.

For most cases, the wavelength spectral distance between the FRA pump wavelengths, presented in TABLES 5-8, are longer than $8 \mathrm{~nm}$. At the closest point it will reach to $2.3 \mathrm{~nm}$. By using narrow spectral width laser diodes at the places where the pump wavelengths are near the adjacent pump wavelengths, the pump powers will not interfere with each other and sound operation is guaranteed. Typical spectral width of a laser diode is about $0.1-10 \mathrm{~nm}$.

FIG. 5 shows a comparison between the convergence speeds of PSO and GA optimization processes which are carried on the optimization of one of the configurations of our WDM systems. It is apparent that PSO acts faster and more efficiently in searching for the optimum values for a system under test.

\section{CONCLUSION}

In this paper, we have used an effective optimization method, called particle swarm optimization (PSO) to offer optimum constructions for hybrid erbium-doped fiber amplifier/ fiber Raman amplifiers (EDFA/FRAs). PSO allowed us to include a high number of parameters in the optimization process, so that we could optimize as much as 24 parameters simultaneously. Using the efficient PSO algorithm, pump wavelengths of FRA are optimized in addition to the FRA pump powers, EDFA pump power and wavelength, $\mathrm{Er}^{3+}$ concentration, and EDFA length. This results in obtaining hybrid amplifiers with gain spectrum variations of as low 
as $1.043 \mathrm{~dB}$ for a $108.5 \mathrm{~km}$ and $1.794 \mathrm{~dB}$ for a $174 \mathrm{~km}$, 10-pumped-FRA hybrid EDFA/FRA, respectively. A comparison between the convergence speeds of PSO and genetic algorithm is done that shows that PSO is faster than genetic algorithm in converging toward the best values of amplifier parameters.

\section{ACKNOWLEDGMENT}

The first author would like to thank the Sepidan Branch of the Islamic Azad University, Shiraz, Iran, for the financial support of this project.

\section{REFERENCES}

1. P. C. Becker, N. A. Olsson, and J. R. Simpson, Erbiumdoped Fiber Amplifiers: Fundamentals and Technology (Academic Press, San Diego, USA, 1999), Chapter 1.

2. M. N. Islam, Raman Amplifiers for Telecommunications I \& II (Springer, New York, USA, 2004).

3. H. Masuda and S. Kawai, "Wide-band and gain-flattened hybrid fiber amplifier consisting of an EDFA and a multiwavelength pumped Raman amplifier," IEEE Photon. Technol. Lett. 11, 647-649 (1999).

4. J. Kennedy and R. C. Eberhart, Swarm Intelligence (Academic Press, San Diego, USA, 2001), Chapter 7.

5. A. P. Engelbrecht, Fundamental of Computational Swarm Intelligence (John Wiley \& Sons, New York, USA, 2005), Chapter 16.

6. A. Mowla and N. Granpayeh, "Optimum design of a hybrid erbium-doped fiber amplifier/fiber Raman amplifier using particle swarm optimization,” Appl. Opt. 48, 979-984 (2009).

7. C. Cheng and M. Xiao, "Optimization of an erbium-doped fiber amplifier with radial effects," Opt. Comm. 254, 215-222 (2005).

8. C. Cheng, Z. Xu, and C. Sui, "A novel design method: a genetic algorithm applied to an erbium-doped fiber amplifier," Opt. Comm. 227, 371-382 (2003).

9. C. Cheng, "A global design of an erbium-doped fiber and an erbium-doped fiber amplifier," Opt. Laser Technol. 36, 607-612 (2004).

10. B. Neto, A. L. J. Teixeira, N. Wada, and P. S. Andre, "Efficient use of hybrid genetic algorithms in the gain optimization of distributed Raman amplifiers," Opt. Express 15, 17520-
17528 (2007).

11. X. Liu, "Optimization for various schemes of distributed fiber Raman amplifiers," J. Opt. A: Pure Appl. Opt. 6, 10171026 (2004).

12. X. Liu and Y. Li, "Optimizing the bandwidth and noise performance of distributed multi-pump Raman amplifiers," Opt. Comm. 230, 425-431 (2004).

13. J. S. Yoon and N. Kim, "Optimization of diffractive optical elements by genetic algorithm," J. Opt. Soc. Korea 4, 30-36 (2000).

14. J. H. Jung, "Optimal design of dielectric-loaded surface plasmon polariton waveguide with genetic algorithm," J. Opt. Soc. Korea 14, 277-281 (2010).

15. C. R. Giles and E. Desurvire, "Modeling erbium-doped fiber amplifiers," IEEE J. Lightwave Technol. 9, 271-283 (1991).

16. A. A. M. Saleh, R. M. Jopson, J. D. Evankow, and J. Aspell, "Modeling of gain in erbium-doped fiber amplifiers," IEEE Photon. Technol. Lett. 2, 714-717 (1990).

17. C. Cheng and M. Xiao, "Optimization of a dual pumped L-band erbium-doped fiber amplifier by genetic algorithm," IEEE J. Lightwave Technol. 24, 3824-3829 (2006).

18. W. J. Miniscalco, "Erbium-doped glasses for fiber amplifiers at $1500 \mathrm{~nm}$," IEEE J. Lightwave Technol. 9, 234-250 (1991).

19. X. Liu and B. Lee, "A fast and stable method for Raman amplifier propagation equations," Opt. Express 11, 2163-2176 (2003).

20. A. Mowla and N. Granpayeh, "A novel design approach for erbium-doped fiber amplifiers by particle swarm optimization," Prog. In Electromagn. Res. M 3, 103-118 (2008).

21. J. Bromage, "Raman amplification for fiber communications systems," IEEE J. Lightwave Technol. 22, 79-93 (2004).

22. X. Liu and B. Lee, "Effective shooting algorithm and its application to fiber amplifiers," Opt. Express 11, 1452-1461 (2003).

23. A. Mowla and N. Granpayeh, "Design of a flat gain multipumped distributed fiber Raman amplifier by particle swarm optimization," J. Opt. Soc. Am. A 25, 3059-3066 (2008).

24. C. Headley and G. P. Agrawal, Raman Amplification in Fiber Optical Communication Systems (Elsevier, Burlington, USA, 2005), pp. 33-163.

25. J. Kennedy and R. C. Eberhart, "Particle swarm optimization," in Proc. IEEE Int. Conf. on Neural Networks (Perth, WA, Australia, 1995), pp. 1942-1948.

26. R. C. Eberhart and J. Kennedy, "A new optimizer using particle swarm theory," in Proc. 6th Int. Symposium on Micro Machine and Human Science (Nagoya, Japan, 1995), pp. 39-43. 